\title{
Are Bundled Payments a Viable Reimbursement Model for Revision Total Joint Arthroplasty?
}

\author{
P. Maxwell Courtney MD, Blair S. Ashley MD, Eric L. Hume MD, \\ Atul F. Kamath MD
}

Received: 9 March 2016/ Accepted: 16 June 2016/Published online: 29 June 2016

(C) The Association of Bone and Joint Surgeons \& 2016

\begin{abstract}
Background Alternative payment models, such as the Centers for Medicare \& Medicaid Services (CMS) Bundled Payment for Care Improvement (BPCI) initiative, aim to decrease overall costs for hip and knee arthroplasties.

Questions/purposes We asked: (1) Is there any difference in the CMS episode-of-care costs, hospital length of stay, and readmission rate from before and after implementation of our bundled-payment program? (2) Is there any difference in reimbursements and resource utilization between revision THA and TKA at our institution? (3) Are there any independent risk factors for patients with high costs who may not be appropriate for a bundled-payment system for revision total joint arthroplasty (TJA)?

Methods Between October 2013 and March 2015, 218 patients underwent revision TKA or THA in one health system. Two hundred seventeen patients were reviewed as part of this study, and one patient with hemophilia was excluded from the analysis as an outlier. Our institution
\end{abstract}

Each author certifies that he or she, or a member of his or her immediate family, has no funding or commercial associations (eg, consultancies, stock ownership, equity interest, patent/licensing arrangements, etc) that might pose a conflict of interest in connection with the submitted article.

All ICMJE Conflict of Interest Forms for authors and Clinical Orthopaedics and Related Research ${ }^{\mathbb{R}}$ editors and board members are on file with the publication and can be viewed on request. Each author certifies that his or her institution approved the human protocol for this investigation, that all investigations were conducted in conformity with ethical principles of research, and that informed consent for participation in the study was obtained.

P. M. Courtney, B. S. Ashley, E. L. Hume, A. F. Kamath ( $\square)$ Department of Orthopaedic Surgery, University of Pennsylvania, 3737 Market Street, 6th Floor, Philadelphia, PA 19104, USA e-mail: atul.kamath@uphs.upenn.edu began a BPCI program for revision TJA during this study period. Patients' procedures done before January 1, 2014 at one hospital and January 1, 2015 at another hospital were not included in the bundled-care arrangement (70 revision TKAs and 56 revision THAs), whereas 50 revision TKAs and 41 revision THAs were performed under the BPCI initiative. Patient demographics, medical comorbidities, episode-of-care reimbursement data derived directly from CMS, length of stay, and readmission proportions were compared between the bundled and nonbundled groups.

Results Length of stay in the group that underwent surgery before the bundled-care arrangement was longer than for patients whose procedures were done under the BPCI (mean 4.02 [SD, 3.0 days] versus mean 5.27 days [SD, 3.6 days]; $p=0.001$ ). Index hospitalization reimbursement for the bundled group was less than for the nonbundled group (mean USD 17,754 [SD, USD 2741] versus mean USD 18,316 [SD, USD 4732]; $\mathrm{p}=0.030$ ). There was no difference, with the numbers available, in total episode-ofcare CMS costs between the two groups (mean USD 38,107 [SD, USD 18,328] versus mean USD 37,851 [SD, USD 17,208]; $p=0.984)$. There was no difference, with the numbers available, in the total episode-of-care CMS costs between revision hip arthroplasties and revision knee arthroplasties (mean USD 38,627 [SD, USD 18,607] versus mean USD 37,414 [SD, USD 16,884]; $\mathrm{p}=0.904$ ). Disposition to rehabilitation (odds ratio [OR], 5.49; 95\% CI, $1.97-15.15 ; \mathrm{p}=0.001)$, length of stay 4 days or greater (OR, 3.66; 95\% CI, 1.60-8.38; $\mathrm{p}=0.002$ ), and readmission within 90 days (OR, 6.99; 95\% CI, 2.58-18.91; $p<0.001)$ were independent risk factors for high-cost episodes.

Conclusions Bundled payments have the potential to be a viable reimbursement model for revision TJA. Owing to the unpredictable nature of the surgical procedures, inherent high risks of complications, and varying degrees of 
surgical complexity, future studies are needed to determine whether bundling patients having revision TJA will result in improved care and decreased costs.

Level of Evidence Level IV, economic and decision analysis.

\section{Introduction}

THAs and TKAs are among the largest and fastest-growing healthcare expenditures [7, 13, 14], totaling USD 6.6 billion in Medicare payments for 2013 alone [4]. While costs of primary total joint arthroplasties (TJA) tend to be fairly predictable, revision TJAs have a greater degree of surgical complexity and inherent risk of complications. As policymakers, hospitals, and insurers attempt to improve cost utilization, alternative-payment models such as bundled payments have increased in popularity, with the hopes of streamlining all costs associated with an episode of care. The Centers for Medicare \& Medicaid Services (CMS) rolled out the Bundled Payment for Care Improvement (BPCI) initiative in 2011 to align incentives among healthcare stakeholders to reduce the cost to Medicare for joint replacements [5]. Although bundled payments for primary joint arthroplasty have been shown to be a feasible alternative to traditional fee-for-service payments [3, 8, 10], it has yet to be determined if bundled payments are appropriate for revision TJA.

Revision hip and knee arthroplasties have added to the increasing costs associated with TJA, owing to the rising number of these procedures being performed [13]. A revision TJA generally incurs greater costs than a primary TJA and is of particular interest, and challenge, when considering how to curb costs through a bundled-payment system [9]. Concerns exist about bundling revision arthroplasty because of a complex and heterogeneous patient population, higher rate of complications, and substantial variability and difficulty in surgical cases. Bundling all postoperative care within 90 day is of particular concern in revisions, because a substantial proportion of readmissions are the result of medical issues unrelated to the index surgical procedure [17].

The purpose of our study was to evaluate the viability of our institution's bundled payment model for revision THA and TKA. We therefore asked: (1) Is there any difference in CMS episode-of-care costs, hospital length of stay, and readmission rate from before and after implementation of our bundled-payment program? (2) Is there any difference in reimbursement and resource utilization between revision THA and TKA at our institution? (3) Are there any independent risk factors for patients with high costs who may not be appropriate for a bundled-payment system for revision TJA?

\section{Patients and Methods}

Between October 2013 and March 2015, 218 patients underwent revision TKA or THA in one health system. Two hundred seventeen patients were reviewed as part of the study, and one patient with hemophilia was excluded from the analysis as a cost outlier. This patient required continuous factor infusion throughout his hospital stay, totaling more than USD 6 million in CMS reimbursement. An exclusion from our bundle was granted by CMS. This study was approved by and conducted according to our institutional review board standards. We queried the health system's financial database for all patients during the study period in the Diagnosis Related Groups (DRG) 466, 467, or 468, corresponding to revision THA and TKA. Diagnosis and type of revision surgery also were noted. Financial data were derived directly from CMS cost data, pulled by our third-party bundle convener. Surgery was performed by one of seven fellowship-trained arthroplasty surgeons at both facilities.

Procedures done before January 1, 2014 at one hospital and January 1, 2015 at another hospital were not included under the bundled-care arrangement (70 revision TKAs and 56 revision THAs), while those done after those dates were performed under the BPCI initiative (50 revision TKAs and 41 revision THAs). Both hospitals are affiliates of the same health system, in the same city, and serve the same patient population. Preoperative medical risk-stratification programs, perioperative pain and rehabilitation protocols, and postdischarge provider networks generally are standardized between campuses. Implant costs are standardized between hospitals. There were 110 patients at the first hospital and 106 at the second hospital. There was no difference, with the numbers available, between patients at either hospital with respect to age (mean 67.5 years [SD, 13.0 years] versus mean 67.4 years [SD, 11.5 years]; $\mathrm{p}=0.916$ ), BMI (mean $32.0 \mathrm{~kg} / \mathrm{m}^{2}\left[\mathrm{SD}, 8.9 \mathrm{~kg} / \mathrm{m}^{2}\right]$ versus mean $31.0 \mathrm{~kg} / \mathrm{m}^{2}$ [SD, $\left.\left.7.4 \mathrm{~kg} / \mathrm{m}^{2}\right] ; \mathrm{p}=0.340\right)$, proportion of female patients (70 of 110 [63\%] versus 56 of 106 [52\%]; $p=0.107$ ), patients having revision hip arthroplasty (48 of 110 [43\%] versus 49 of 106 [46\%]; $\mathrm{p}=0.702)$, minority patients $(29$ of 110 [26\%] versus 29 of 106 [27\%]; p = 0.869), patients residing in a ZIP code of bottom quartile of median household income (20 of 110 [18\%] versus 19 of 106 [17\%]; $\mathrm{p}=0.960$ ), or patients with a Charlson Comorbidity Index of 3 or greater (23 of 110 [20\%] versus 17 of 106 [15\%]; $\mathrm{p}=0.356$ ).

The BPCI included all costs associated with the index hospital admission and all care up to 90 days postdischarge. CMS cost data included anchor inpatient admission, postdischarge care (skilled nursing facility, inpatient rehabilitation, or home health care), Medicare Part B payments, readmission reimbursement, and durable 
medical equipment. Implementation of the BPCI at both hospitals included education of attending surgeons, orthopaedic floor nurses, physical therapists, social workers, and residents to streamline care for patients undergoing revision joint arthroplasty. Emphasis was placed on reducing length of stay through early rehabilitation and standardized pain protocols. Patients were educated preoperatively on the benefits of disposition to home postoperatively. Improved communication with subacute facilities and the emergency room helped minimize readmissions. Patients enrolled in the bundled-payment initiative were flagged in the electronic medical record to alert providers when considering discharge or readmission to the hospital.

We collected cost data, patient demographics, comorbidities, length of stay data, discharge disposition, and 90day readmission rates for all patients in the study. Medical comorbidities included in the Charlson Comorbidity Index were noted from the preoperative history and physical examination. The Charlson Comorbidity Index is a measure of a patient's physical health status and has been validated [6]. Discharge disposition from the index hospital admission was classified as home, home with home health assistance, skilled nursing facility, or inpatient rehabilitation facility. In-hospital complications were defined according to a previously published classification and definition [18]. As a measure of socioeconomic status, the patient's zip code of residence was documented and paired with each zip code's median household income according to the US Census Bureau [20]. Patients residing in a zip code representative of the bottom quartile of zip code median household income were defined as being of low socioeconomic status. To normalize costs across insurance carriers, cost data reported to our database included payments to the provider for services at Medicare rates.

The total episode-of-care cost was defined as all costs for the patient from the time of surgery through 90 days postdischarge of the index hospital admission. Inpatient costs included the index hospital admission. Rehabilitation costs were defined as all payments to a skilled nursing facility or inpatient rehabilitation facility. Part B costs refer to the portion of the Medicare Severity-DRG care that would be paid under Medicare Part B and represent outpatient payments, and laboratory and radiology charges.

Of the 216 patients in the study cohort, there were 91 in the health system's bundled-payment initiative (42\%) before the start date of the bundle at each institution. Of the 97 revision THAs performed, 35 (36\%) were both-component revisions, 12 (12\%) were isolated femoral component revisions, 36 (37\%) were isolated acetabular revisions, and $14(14 \%)$ were ball head and liner exchanges. Of the 119 revision TKAs, 97 (82\%) were bothcomponent revisions, 15 (13\%) were single-component revisions, and seven $(6 \%)$ were polyethylene exchanges.
The most common indication for revision was aseptic loosening ( 81 of 216 patients; $38 \%$ ), followed by instability (63 of 216 patients; 29\%), and infection (49 of 216 patients; $23 \%$ ). There were no differences in the types of revision THAs $(\mathrm{p}=0.925)$ or TKAs $(\mathrm{p}=0.549)$ between the bundled and nonbundled groups. The remaining 125 patients were billed according to the traditional fee-forservice model $(58 \%)$. The mean age for all patients was 67.5 years (SD, 12.3 years). There were $97(45 \%)$ revision hip arthroplasties and 119 (55\%) revision knee arthroplasties. The mean length of stay was 4.74 days (SD, 3.4 days). Eighty patients (37\%) were discharged to home with or without home health assistance. The mean episode-ofcare cost for all patients was USD 37,959 (SD, USD 17, 647 ) and the mean Part B charge was USD 5405 (SD, USD 2837) (Table 1). There were 29 (13\%) readmissions to the hospital within 90 days. The most common reason for readmission was a wound complication in 18 patients.

Table 1. Descriptive statistics of all patients in the study population

\begin{tabular}{|c|c|}
\hline Demographic data $(\mathrm{n}=216)$ & Value \\
\hline Mean age (years) & $67.5 \pm 12.3$ \\
\hline \multicolumn{2}{|l|}{ Gender $(\%)$} \\
\hline Female & $126(58)$ \\
\hline Male & $90(42)$ \\
\hline \multicolumn{2}{|l|}{ Ethnicity (\%) } \\
\hline White & $157(73)$ \\
\hline Nonwhite & $59(27)$ \\
\hline \multicolumn{2}{|l|}{ Surgery $(\%)$} \\
\hline Revision knee arthroplasty & $119(55)$ \\
\hline Revision hip arthroplasty & $97(45)$ \\
\hline Mean BMI $\left(\mathrm{kg} / \mathrm{m}^{2}\right)$ & $31.5 \pm 8.2$ \\
\hline Mean length of stay (days) & $4.74 \pm 3.4$ \\
\hline Bottom quartile of median household income $(\%)$ & $39(18)$ \\
\hline Mean episode of care costs (USD) & $37,959 \pm 17,647$ \\
\hline Mean index inpatient admission costs (USD) & $18,079 \pm 4017$ \\
\hline Mean postdischarge rehabilitation costs (USD) & $8948 \pm 11,313$ \\
\hline Mean home health costs (USD) & $2426 \pm 1707$ \\
\hline Mean postdischarge outpatient care (USD) & $819 \pm 1243$ \\
\hline $\begin{array}{l}\text { Mean Charlson Comorbidity Index } \\
\text { of } 3 \text { or greater }(\%)\end{array}$ & $\begin{array}{l}1.37 \pm 1.83 \\
40(19)\end{array}$ \\
\hline In-hospital complication (\%) & $60(28)$ \\
\hline \multicolumn{2}{|l|}{ Discharge disposition (\%) } \\
\hline Home & $80(37)$ \\
\hline Skilled nursing or rehabilitation facility & $136(63)$ \\
\hline Readmission within 90 days (\%) & $29(13)$ \\
\hline
\end{tabular}


Table 2. Data comparison for bundled and nonbundled groups

\begin{tabular}{|c|c|c|c|}
\hline Patient characteristic & $\begin{array}{l}\text { Bundled } \\
\text { payment } \\
\text { group } \\
(\mathrm{n}=91)\end{array}$ & $\begin{array}{l}\text { Nonbundled } \\
\text { payment } \\
\text { group } \\
(\mathrm{n}=125)\end{array}$ & $\mathrm{p}$ value \\
\hline Mean age (years) & $67.0 \pm 11.4$ & $67.9 \pm 13.0$ & 0.373 \\
\hline \multicolumn{4}{|l|}{ Gender $(\%)$} \\
\hline Female & 45 (49) & $81(64)$ & \multirow[t]{2}{*}{0.034} \\
\hline Male & $46(51)$ & $44(36)$ & \\
\hline \multicolumn{4}{|l|}{ Ethnicity (\%) } \\
\hline White & $68(75)$ & $89(71)$ & \multirow[t]{2}{*}{0.671} \\
\hline Nonwhite & $23(25)$ & $36(29)$ & \\
\hline \multicolumn{4}{|l|}{ Surgery $(\%)$} \\
\hline Revision knee arthroplasty & $50(55)$ & $74(55)$ & \multirow[t]{2}{*}{0.920} \\
\hline Revision hip arthroplasty & $41(45)$ & $56(45)$ & \\
\hline Mean body mass index $\left(\mathrm{kg} / \mathrm{m}^{2}\right)$ & $31.6 \pm 7.5$ & $31.5 \pm 8.7$ & 0.810 \\
\hline Mean length of stay (days) & $4.0 \pm 3.0$ & $5.3 \pm 3.6$ & 0.001 \\
\hline $\begin{array}{c}\text { Bottom quartile of median } \\
\text { household income }(\%)\end{array}$ & $14(15)$ & $25(20)$ & 0.512 \\
\hline $\begin{array}{l}\text { Mean episode of care costs } \\
\text { (USD) }\end{array}$ & $\begin{array}{r}38,107 \pm \\
18,328\end{array}$ & $\begin{array}{r}37,851 \pm \\
17,208\end{array}$ & 0.984 \\
\hline $\begin{array}{l}\text { Mean index inpatient } \\
\text { admission costs (USD) }\end{array}$ & $\begin{array}{c}17,754 \pm \\
2741\end{array}$ & $\begin{array}{c}18,316 \pm \\
4732\end{array}$ & 0.030 \\
\hline $\begin{array}{l}\text { Mean postdischarge } \\
\quad \text { rehabilitation costs (USD) }\end{array}$ & $9871 \pm 12,174$ & $\begin{array}{l}8276 \pm \\
10,642\end{array}$ & 0.347 \\
\hline Mean home health costs (USD) & $2465 \pm 1854$ & $\begin{array}{r}2398 \pm \\
1598\end{array}$ & 0.764 \\
\hline $\begin{array}{l}\text { Mean postdischarge outpatient } \\
\text { care (USD) }\end{array}$ & $911 \pm 1456$ & $751 \pm 1063$ & 0.395 \\
\hline $\begin{array}{l}\text { Mean Charlson Comorbidity } \\
\text { Index }\end{array}$ & $1.08 \pm 1.46$ & $1.57 \pm 2.03$ & 0.114 \\
\hline of 3 or greater $(\%)$ & $15(16)$ & $25(20)$ & 0.631 \\
\hline In-hospital complication (\%) & $24(26)$ & $36(29)$ & 0.810 \\
\hline \multicolumn{4}{|l|}{ Discharge disposition (\%) } \\
\hline Home & $32(35)$ & $48(38)$ & \multirow[t]{2}{*}{0.729} \\
\hline $\begin{array}{l}\text { Skilled nursing or } \\
\text { rehabilitation facility }\end{array}$ & $59(65)$ & $77(62)$ & \\
\hline
\end{tabular}

\section{Statistical Analysis}

An a priori power analysis was first performed to determine the appropriate sample size for our primary outcome measure of total episode-of-care costs between the bundled and nonbundled groups. Because pilot data from our institution were not available for CMS reimbursements for revision TJA, we chose a standard moderate effect size (Cohen's $d=0.5$ ), detecting a 33\% overlap between the two groups [19]. Assuming a Type I error rate of 0.05 , at least 64 patients were needed in each group to achieve a power of 0.80. Descriptive statistics were calculated for all patients in the study population (Table 1). Continuous variables then were tested for normality using the Shapiro-
Wilk test and analyzed using the Mann-Whitney U test, because they were not normally distributed. Categorical data were analyzed with a chi-square test to determine any difference between the bundled and nonbundled groups (Table 2). To control for confounding variables, a multivariate logistic regression analysis was performed to identify independent risk factors for patients with high costs, defined as falling in the upper quartile of costs (greater than USD 44,564). Statistical significance for all tests was set at 0.05 . Statistical analysis was performed using Excel ${ }^{\mathbb{R}}$ (Microsoft ${ }^{\circledR}$ Corporation, Redmond, WA, USA) and SPSS version 22.0 (IBM, Armonk, NY, USA).

\section{Results}

We found no difference, with the numbers available, in total episode-of-care costs between the bundled-payment group and the nonbundled group (mean USD 38,107 [SD, USD 18,328] versus USD 37,851 [SD, USD 17,208]; $\mathrm{p}=$ 0.984 ) (Fig. 1). There was no difference, with the numbers available, in the rate of discharge to a rehabilitation facility (59 of 91 patients [65\%] versus 77 of 125 patients [62\%]; $\mathrm{p}=0.729$ ) or costs of postdischarge rehabilitation (mean USD 9871 [SD, USD 12,174] versus USD 8276 [SD, USD $10,642] ; p=0.347$ ) (Table 2). The bundled group had a shorter length of stay (mean 4.02 days [SD, 3.0 days] versus mean 5.27 days $[\mathrm{SD}, 3.6$ days]; $\mathrm{p}=0.001$ ) and smaller index hospitalization costs than the nonbundled group (mean USD 17,754 [SD, USD 2741] versus USD 18,316 [SD, USD 4732]; $\mathrm{p}=0.030$ ). With the numbers available, there was no difference in 90-day readmission rates (17of 91 patients [18\%] versus 12 of 125 patients [10\%]; $\mathrm{p}=0.083)$. Twenty-six of 91 patients $(29 \%)$ and 40 of $125(32 \%)$ were discharged with home health care and six of $91(7 \%)$ and eight of $125(6 \%)$ were discharged home without care in the bundled and nonbundled groups, respectively, with no difference in home health cost between the groups (mean USD 2465 [SD, USD 1854] versus USD 2398 [SD, USD 1598]; $p=0.764$ ). For patients who were readmitted to the hospital within 90 days, the mean readmission cost was USD 15,978 (SD, USD 5642).

There was no difference, with the numbers available, in the total episode-of-care costs between revision hip arthroplasties and revision knee arthroplasties (mean USD 38,627 [SD, USD 18,607] versus mean USD 37,414 [SD, USD 16,884]; $p=0.904)$. Similarly, there was no difference in length of stay (mean 5.25 days [SD, 4.32 days] versus mean 4.32 days [SD, 2.42 days]; $p=0.170$ ), discharge disposition to rehabilitation (63 of 97 patients [65\%] versus 73 of 119 patients [61\%]; $p=0.585$ ), and 90day readmission rate (12 of 97 patients [12\%] versus 17 of 119 patients [14\%]; $\mathrm{p}=0.681$ ). The bundled-payment and 
Fig. 1A-B The distribution of costs between the (A) bundled and (B) nonbundled revision arthroplasty groups are shown. Although the bundled group had lower index inpatient admission costs $(\mathrm{p}=0.030)$, there was no statistical difference in overall costs. DME = durable medical equipment.
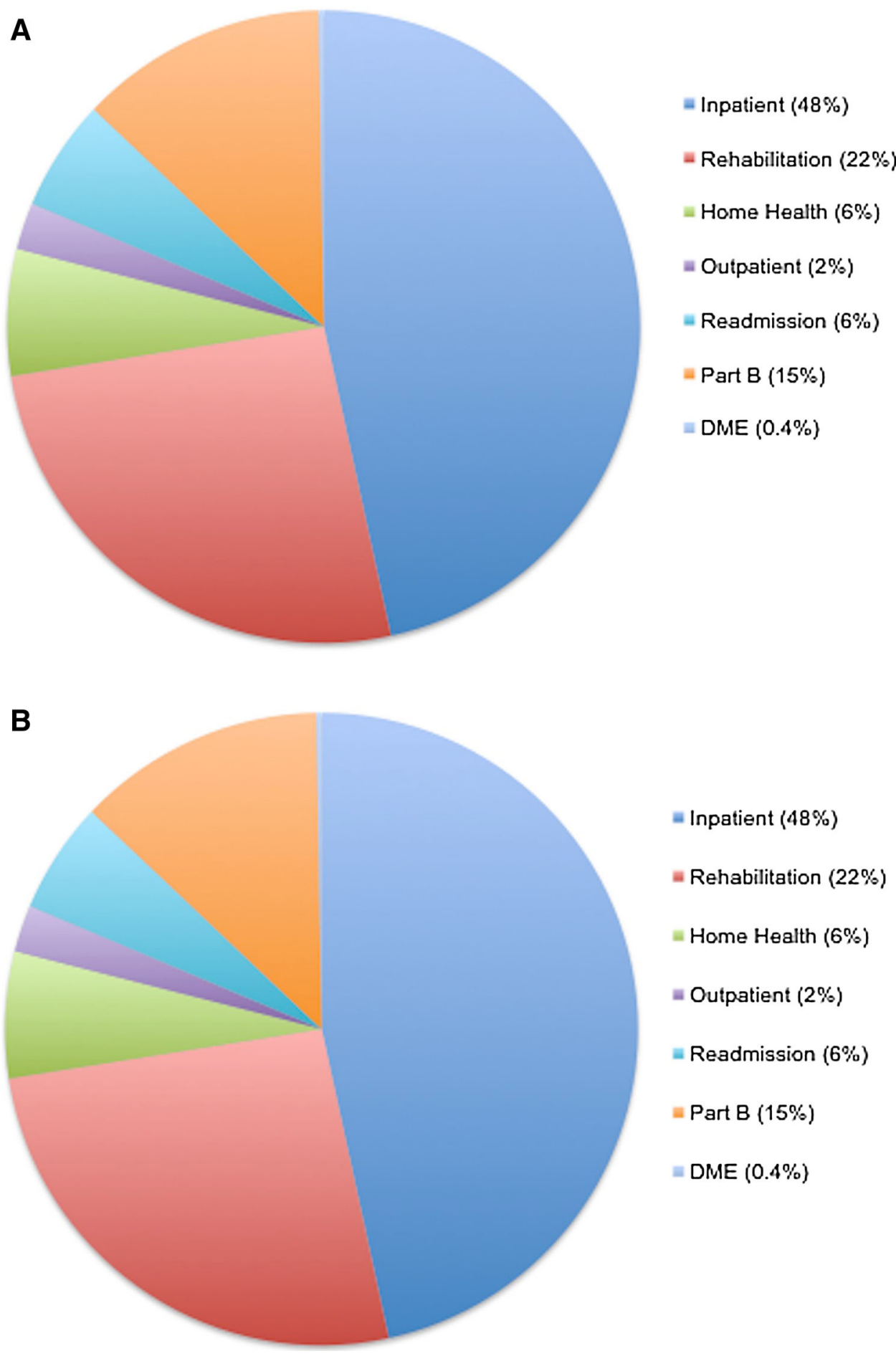

nonbundled-payment groups had comparable rates of hip arthroplasties (41 of 91 patients [45.1\%] versus 56 of 125 patients [44.8\%]; $\mathrm{p}=0.920)$. There also was no difference in mean episode-of-care costs between the bundled and nonbundled groups for revision hip (mean USD 40,679 [SD, USD 18,945] versus mean USD 37,124 [SD, USD $18,381] ; \mathrm{p}=0.322$ ) or revision knee arthroplasty (mean
USD 35,997 [SD, USD 17,718] versus mean USD 38,441 [SD, USD 16,307]; $\mathrm{p}=0.317)$. The bundled-payment group had lower Medicare Part B costs than the nonbundled group (mean USD 4836 [SD, USD 2722] versus mean USD 5820 [SD, USD 2858]; $\mathrm{p}=0.011$ ).

Independent risk factors for episode-of-care costs in the upper quartile of our study group (greater than USD 
Table 3. Multivariate logistic regression analysis of risk factors for episode-of-care costs in the upper quartile (greater than USD 44,564)

\begin{tabular}{llll}
\hline Patient risk factor & $\begin{array}{l}\text { Odds } \\
\text { ratio }\end{array}$ & $95 \%$ CI & p value \\
\hline Female gender & 1.58 & $0.70-3.57$ & 0.270 \\
Bottom quartile household income & 0.78 & $0.26-2.33$ & 0.667 \\
Age older than 75 years & 1.24 & $0.52-2.92$ & 0.618 \\
Disposition to skilled nursing & 5.49 & $1.97-15.15$ & 0.001 \\
$\quad$ facility or rehabilitation & & & \\
BMI > 35 kg/m & 1.22 & $0.53-2.81$ & 0.639 \\
Revision hip arthroplasty & 0.85 & $0.39-1.86$ & 0.689 \\
Charlson Comorbidity Index score & 0.81 & $0.32-2.07$ & 0.665 \\
$\quad$ of 3 or greater & & & \\
Nonwhite ethnicity & 1.18 & $0.45-3.08$ & 0.723 \\
In-hospital complication & 1.79 & $0.80-3.99$ & 0.153 \\
Length of stay 4 days or greater & 3.66 & $1.60-8.38$ & 0.002 \\
90-day readmission & 6.99 & $2.58-18.91$ & $<0.001$ \\
\hline
\end{tabular}

44,564) included disposition to a skilled nursing or rehabilitation facility (odds ratio [OR], 5.49; 95\% CI, $1.97-$ $15.15 ; \mathrm{p}=0.001$ ) (Table 3), length of stay 4 days or greater (OR, 3.66; 95\% CI, 1.60-8.38; $\mathrm{p}=0.002$ ), and readmission within 90 days (OR, 6.99; 95\% CI, 2.58-18.91; $\mathrm{p}<0.001$ ). Age older than 75 years, nonwhite ethnicity, Charlson Comorbidity Index score of 3 or greater, obesity, and low socioeconomic status were not associated with increased episode-of-care costs in our study.

\section{Discussion}

Several studies have shown the effectiveness of bundledpayment models for primary hip and knee arthroplasties $[3,8,10]$. However, revision TJA presents a complex set of problems for bundling episode-of-care payments. Because of the inherent higher risk of complications, unpredictable nature of the surgical procedures, and varying degrees of technical difficulty, surgeons and hospitals have reservations about expanding the CMS bundled-payment initiative framework to include revision hip and knee arthroplasties. Revision TJAs have a higher cost burden than primary TJAs $[2,16]$, and a few high-cost outliers can severely reduce or even eliminate the hospital's margin on the bundle [15]. We sought to determine whether there were any differences in CMS episode-of-care costs, hospital length of stay, and readmission rate from before and after implementation of our bundled payment program; if there were any differences in reimbursements and resource utilization between revision THAs and TKAs at our institution; and if there were any independent risk factors for patients with high costs who may not be appropriate for a bundled-payment system for revision TJA?
Our study has several limitations. Our cost data included only CMS episode-of-care reimbursement, not the true cost to the hospital or surgeon for each patient. Accurately identifying true costs is difficult to measure, and hospitals may be financially disadvantaged if they enter a bundledpayment arrangement without knowing the true costs for a procedure. We included all patients undergoing revision THAs or TKAs in our analysis, except for one patient with hemophilia. This patient required continuous factor infusion and had a USD 6 million episode-of-care CMS cost. He was excluded from the BPCI after a formal appeal to CMS. Tertiary-care hospitals such as ours will face challenges treating these medically complex patients who can influence bundled-payment arrangement results. Implant costs vary tremendously between an upsized primary and a modular revision component. Perhaps even a single, difficult revision can ruin a bundled-payment arrangement. With more data, payers and policymakers should distinguish between the degree of difficulty and variability of the risk of complications of each revision procedure to fairly compensate and protect surgeons and hospitals. Although powered to detect a modest difference in costs between the bundled and nonbundled groups, the risk of committing a Type II error when interpreting results from differences in the hip and knee subgroups is high. We also included data from two hospitals in our health system, which may have variability in discharge planning and readmission. However, our department attempted to streamline the disposition process for surgeons, social workers, and therapists and raise flags in the electronic medical record for emergency medicine physicians considering readmission at either hospital. Likewise, attempts at standardization protocols have been in place for care pathways at both hospitals. Patient demographics and procedures were comparable between the two hospitals, helping to minimize bias.

The bundled and nonbundled groups had markedly lower costs for revision TJA than the reported mean for a large group of private payers [16]. This is attributable to the standardization of our cost numbers to CMS rates, which traditionally has lower reimbursement than private insurers. The percentage breakdowns, however, were comparable to those of other studies [3, 10]. Bozic et al. [3] reported that postdischarge care accounted for $36 \%$ of payments including all primary and revision arthroplasties (DRG 462, 466-470), whereas $37.7 \%$ of episode-of-care costs in our series were for postdischarge care. Although we found no difference in total episode-of-care reimbursements between the bundled and nonbundled groups, our institution's BPCI was associated with a decrease in the length of stay, which may translate to lower index hospitalization costs than for the nonbundled group. While other confounding factors can contribute to a decrease in length 
of stay, the lack of an observed difference in costs is likely representative of the complex and heterogeneous population undergoing revision arthroplasties, and not necessarily a failure of our bundled-payment initiative. A higher readmission rate may be a direct result of squeezing length of stay [7]. The optimal length of stay to minimize 90-day readmissions among patients undergoing TJAs is a complex problem and has yet to be reported in the literature. A recent survey of members of the American Association of Hip and Knee Surgeons [11] showed concern among arthroplasty surgeons regarding access to care in bundledpayment models, because physicians and hospitals may have a disincentive to operate on more medically complex patients. Although our data showed no socioeconomic or demographic bias, further study is needed to determine whether patients of lower socioeconomic status or those with multiple medical comorbidities will face barriers to obtaining revision arthroplasties in the era of alternative payment models.

We found no difference in the costs of revision THA compared with revision TKA. We also found no difference in the readmission rates or percentage of patients discharged to a rehabilitation facility. This contrasts with another study, which showed a higher clinical and economic burden for revision hip arthroplasty than revision knee arthroplasty [1]. This result should be interpreted with caution owing to the small sample size for this subgroup analysis, because one cost outlier could substantially affect the results. Our calculation of cost data also was different, as we only looked at direct costs to CMS, not overall insurance reimbursement.

We found that length of stay 4 days or greater, disposition to a rehabilitation facility, and 90-day readmission are risk factors for being cost outliers in patients undergoing revision TJAs. Although these results are not surprising, it is interesting that age older than 75 years, nonwhite ethnicity, Charlson Comorbidity Index score of 3 or greater, obesity, and low socioeconomic status were not associated with increased episode-of-care costs. Our results are in agreement with those of one large series [16] that showed patients who were discharged to a skilled nursing facility and experienced readmission had substantially higher costs of care with revision TJA. A recent study looking at risk factors for readmission and poor outcomes after revision TJA identified advanced age, obesity, and medically complex patients as risk factors for readmission and extended length of stay [12]. More data are needed in assisting surgeons and hospitals to select appropriate patients for alternative payment models in revision TJA.

The start of a BPCI for revision TJA at our institution was associated with a decrease in hospital length of stay but no difference in episode-of-care costs. Owing to the unpredictable nature of the surgical procedures, inherent high risks of complications, and varying degrees of surgical complexity, future studies are needed to determine whether bundling patients undergoing revision TJAs will result in improved care and decreased costs.

\section{References}

1. Bozic KJ, Kamath AF, Ong K, Lau E, Kurtz S, Chan V, Vail TP, Rubash H, Berry DJ. Comparative epidemiology of revision arthroplasty: failed THA poses greater clinical and economic burdens than failed TKA. Clin Orthop Relat Res. 2015;473:21312138.

2. Bozic KJ, Katz P, Cisternas M, Ono L, Ries MD, Showstack J. Hospital resource utilization for primary and revision total hip arthroplasty. J Bone Joint Surg Am. 2005;87:570-576.

3. Bozic KJ, Ward L, Vail TP, Maze M. Bundled payments in total joint arthroplasty: targeting opportunities for quality improvement and cost reduction. Clin Orthop Relat Res. 2014;472:188193.

4. Centers for Medicare \& Medicaid Services. New Medicare data available to increase transparency on hospital utilization. Available at: https://www.cms.gov/Newsroom/MediaReleaseDatabase/ Fact-sheets/2015-Fact-sheets-items/2015-06-01.html. Accessed February 1, 2016

5. Centers for Medicare \& Medicaid Services. Bundled Payments for Care Improvement (BPCI) initiative: general information. Available at: https://innovation.cms.gov/initiatives/bundled-payments/. Accessed February 1, 2016

6. Charlson ME, Pompei P, Ales KL, MacKenzie CR. A new method of classifying prognostic comorbidity in longitudinal studies: development and validation. J Chronic Dis 1987;40:373-383.

7. Cram P, Lu X, Kates SL, Singh JA, Li Y, Wolf BR. Total knee arthroplasty volume, utilization, and outcomes among Medicare beneficiaries, 1991-2010. JAMA. 2012;308:1227-1236.

8. Froemke CC, Wang L, DeHart ML, Williamson RK, Ko LM, Duwelius PJ. Standardizing care and improving quality under a bundled payment initiative for total joint arthroplasty. J Arthroplasty. 2015;30:1676-1682.

9. Healy WL, Ayers ME, Iorio R, Patch DA, Appleby D, Pfeifer BA. Impact of a clinical pathway and implant standardization on total hip arthroplasty: a clinical and economic study of short-term patient outcome. J Arthroplasty 1998;13:266-276.

10. Iorio R, Clair AJ, Inneh IA, Slover JD, Bosco JA, Zuckerman JD. Early results of Medicare's bundled payment initiative for a 90day total joint arthroplasty episode of care. J Arthroplasty. 2016;31:343-350.

11. Kamath AF, Courtney PM, Bozic KJ, Mehta S, Parsley BS, Froimson MI. Bundled payment in total joint care: Survey of AAHKS membership attitudes and experience with alternative payment models. J Arthroplasty. 2015;30:2045-2056.

12. Keswani A, Lovy AJ, Robinson J, Levy R, Chen D, Moucha CS. Risk factors predict increased length of stay and readmission rates in revision joint arthroplasty. $J$ Arthroplasty. 2016;31:603-608.

13. Kurtz SM, Ong K, Lau E, Mowat F, Halpern M. Projections of primary and revision hip and knee arthroplasty in the United States from 2005 to 2030. J Bone Joint Surg Am. 2007;89: 780-785.

14. Losina E, Walensky RP, Kessler CL, Emrani PS, Reichmann WM, Wright EA, Holt HL, Solomon DH, Yelin E, Paltiel AD, Katz JN. Cost-effectiveness of total knee arthroplasty in the United States: patient risk and hospital volume. Arch Intern Med. 2009;169:1113-1121; discussion 1121-1122. 
15. Mechanic RE. Mandatory Medicare bundled payment: is it ready for prime Time? N Engl J Med. 2015;373:1291-1293.

16. Nichols CI, Vose JG. Clinical outcomes and costs within 90 days of primary or revision total joint arthroplasty. J Arthroplasty. 2016 Jan 21. [Epub ahead of print]

17. Saucedo JM, Marecek GS, Wanke TR, Lee J, Stulberg SD, Puri L. Understanding readmission after primary total hip and knee arthroplasty: who's at risk? J Arthroplasty. 2014;29:256-260.

18. Sink EL, Leunig M, Zaltz I, Gilbert JC, Clohisy J; Academic Network for Conservational Hip Outcomes Research Group.
Reliability of a complication classification system for orthopaedic surgery. Clin Orthop Relat Res. 2012;470:2220-2226.

19. Sullivan GM, Feinn R. Using effect size—or why the $\mathrm{p}$ value is not enough. J Grad Med Educ. 2012;4:279-282.

20. United States Census Bureau. 2014 ACS 5-year estimate: median household income in the past 12 months by 5 digit ZIP code tabulation area. Available at: http://factfinder.census.gov/ faces/nav/jsf/pages/guided_search.xhtml. Accessed February 1, 2016. 